\title{
Influência dos processos de recuperação do solo após mineração de carvão sobre a mesofauna edáfica em Lauro Müller, Santa Catarina, Brasil
}

\author{
Luís Carlos Iuñes de Oliveira Filho ${ }^{1 *}$ \\ Dilmar Baretta ${ }^{2}$ \\ Julio Cesar Pires Santos ${ }^{1}$ \\ ${ }^{1}$ Centro de Ciências Agroveterinárias, Universidade do Estado de Santa Catarina \\ Avenida Luiz de Camões, 2090, CEP 88.520-000, Lages - SC, Brasil \\ ${ }^{2}$ Centro de Educação Superior do Oeste, Universidade do Estado de Santa Catarina \\ Rua Beloni Trombeta Zanin, 68E, CEP 89.8115-630, Chapecó - SC, Brasil \\ * Autor para correspondência \\ iunes1981@gmail.com
}

Submetido em 06/06/2013

Aceito para publicação em 10/02/2014

\section{Resumo}

A degradação do solo devido às atividades antrópicas reduz a densidade e diversidade da fauna edáfica, além de influenciar no processo de decomposição da matéria orgânica. O objetivo deste estudo foi avaliar o efeito da recuperação do solo após a mineração de carvão sobre os grupos da fauna edáfica ao longo do tempo, usando a mata como controle na região de Lauro Müller-SC. Foram utilizadas três áreas antropizadas, duas que passaram por processo de construção do solo após a mineração de carvão (Mina A3 e Mina A5), uma de campo naturalizado e mata nativa usada como controle. A fauna do solo foi coletada utilizando-se um cilindro metálico de $17 \mathrm{~cm}$ de diâmetro, com profundidade de $5 \mathrm{~cm}$, e a extração realizada pelo método de Berlese modificado, seguido da identificação dos organismos. A densidade e a riqueza de grupos foram submetidas à análise de variância. Também se realizou uma análise multivariada dos dados para diferenciação das áreas por meio da análise de curva de resposta principal (PRC). No total foram encontrados 19 táxons, sendo que Acari, Collembola e Formicidae apresentaram as maiores densidades. A PRC evidenciou diferenças entre a mata nativa e pelo menos uma das áreas estudadas em todas as épocas.

Palavras-chave: Análise multivariada; Bioindicadores; Fauna edáfica

\section{Abstract}

Influence of soil reclamation processes after coal mining on the edaphic mesofauna in Lauro Müller, Santa Catarina, Brazil. Soil degradation due to anthropogenic activities reduces the density and diversity of edaphic fauna, besides influencing the organic matter decomposition process. This study aimed to evaluate the effect of soil reclamation after coal mining on the edaphic fauna groups over time, using the forest as control in the region of Lauro Müller, Santa Catarina, Brazil. We used 3 areas with anthropogenic activities, 2 that went through a soil construction process after coal mining (Mine A3 and Mine A5), 1 with naturalized field and indigenous forest used as control. Soil mesofauna was collected using a metal cylinder with $17 \mathrm{~cm}$ in diameter, with depth $5 \mathrm{~cm}$, and the extraction conducted by means of the modified Berlese method, followed 
by the identification of organisms. Group density and richness underwent variance analysis. We also conducted a multivariate data analysis to distinguish the areas by analyzing the principal response curve (PRC). A total of 19 taxa were found, and Acari, Collembola, and Formicidae showed the highest densities. PRC had differences between the indigenous forest and at least one area under study at all times.

Key words: Bioindicators; Edaphic fauna; Multivariate analysis

\section{Introdução}

Um dos maiores passivos ambientais da mineração brasileira situa-se no sul do Estado de Santa Catarina (cerca de 5.100 ha de solos férteis afetados), devido ao longo histórico da mineração de carvão (CASTILHOS; FERNANDES, 2011). A mineração a céu aberto provoca sérios impactos ambientais através da remoção do solo e da cobertura vegetal, além da diminuição das comunidades de organismos do solo (ZEPPELINI et al., 2009). Os processos de mineração tendem a alterar radicalmente os solos, bem como o ambiente onde ocorrem e o seu entorno (ALBUQUERQUE et al., 2011).

O estudo de atributos biológicos como indicadores da qualidade do solo é muito importante para entender os processos ecológicos que ocorrem nestas áreas visando sua recuperação/restauração. A fauna do solo desempenha um papel-chave nos serviços do solo que pode impulsionar o sucesso de recuperação/restauração (SCHON et al., 2012). Estes incluem a ciclagem de nutrientes, aeração e estrutura do solo, produtividade das plantas, ativação da biomassa microbiana, dispersão de sementes e esporos ou controle de pragas de insetos, fontes de alimentos para outros organismos (NICHOLS; NICHOLS, 2003; FROUZ et al., 2006; CARDOSO et al., 2011).

Esses invertebrados podem ser explorados nos processos de remediação e recuperação, devido sua contribuição nas modificações do ambiente, que resultam na criação de micro-habitats e nichos, possibilitando o aumento da biodiversidade (CORREIA, 2002).

Há poucos trabalhos utilizando a fauna invertebrada como indicadora da qualidade do solo para uso no monitoramento de áreas afetadas pela mineração, o que dificulta o seu manejo e recuperação. Assim, o objetivo deste estudo foi avaliar o efeito da recuperação do solo após a mineração de carvão sobre os grupos da fauna edáfica ao longo do tempo, usando a mata como controle na região de Lauro Müller/SC.

\section{Material e Métodos}

O trabalho foi realizado em duas áreas de mineração de carvão a céu aberto, cujo solo passou por processo de construção, no município de Lauro Müller/SC, dentre estas estão às áreas da Mina A3 e Mina A5. Ainda, foram coletadas amostras em uma área de campo naturalizado e uma de mata nativa, que serviu como controle, para efeito de comparação. Nessas áreas, foram realizadas amostragens do solo + serapilheira em quatro épocas: outubro de 2009, fevereiro, maio e agosto de 2010.

O clima da região é definido como mesotérmico úmido com verões quentes (Cfa; segundo a classificação de Köppen), com precipitação e temperatura média anual respectivamente de $1.400 \mathrm{~mm}$ e $19^{\circ} \mathrm{C}$ (LUNARDI NETO et al., 2008). A geologia local é constituída por rochas sedimentares das formações gonduânicas da bacia do Paraná, representadas localmente pelas formações Palermo e Rio Bonito, Grupo Guatá, especificamente a camada minerada Barro Branco (LUNARDI NETO et al., 2008). De acordo com Campos et al. (2003) que analisaram um solo adjacente à área Mina do Apertado, o solo é classificado como Argissolo Vermelho-Amarelo Alítico típico (EMBRAPA, 2006), sendo a classe de solo predominante na área de estudo.

A seguir são apresentadas as práticas de manejo adotadas nas áreas de minas e descrição da Mata Nativa e do Campo Naturalizado. A Mina A3 (A3; 28²1'53.65”S, $\left.49^{\circ} 25^{\prime} 50.62^{\prime} \mathrm{W}\right)$, apresenta cerca de 3,0 ha, a qual após o processo de mineração a céu aberto deu-se início à recuperação da área, com o preenchimento da cava com rejeito piritoso e recobrimento deste com regolito argiloso. Para cobrir os resíduos da mineração utilizouse solo advindo de área de empréstimo e realizado a 
conformação do terreno e plantio de gramíneas. Essa área foi utilizada para fins agrícolas sendo cultivada com aveia (Avena sativa) e fumo (Nicotiana tabacum) e apareceram algumas plantas espontâneas, como picãopreto (Bidens pilosa), junquinho (Cyperus difformis), caruru (Amaranthus sp.). Em decorrência da dificuldade de obtenção do histórico completo da área, não há informações sobre a quantidade de corretivos e adubos aplicados.

A Mina A5 (A5; 2821' 59.55’'S, 49²5' 55.26”'W), apresenta cerca de 5,0 ha, é considerada modelo ideal de recuperação após mineração, sendo construída em 2006, utilizando sólum proveniente de área de empréstimo, regolito argiloso e rocha e plantio de gramíneas em 2009 (CAMPOS et al., 2003). De acordo com o Plano de Recuperação de Áreas Degradadas (PRAD) fornecido pela empresa mineradora, foi aplicada em superfície cama de aviário curtida (quantidade não informada) e calcário dolomítico (4.000 kg.ha-1) e, posteriormente, plantio de Brachiaria decumbens (ANDREOLA, 2011). Nas épocas de coleta, observou-se que a área estava coberta de forma parcialmente homogênea. Maiores informações podem ser obtidas em Andreola (2011).

A Mata Nativa $\left(\mathrm{MN} ; 28^{\circ} 21,50.80 " \mathrm{~S}\right.$, $\left.49^{\circ} 27^{\prime} 25.48^{\prime \prime} \mathrm{W}\right)$, apresenta cerca de 4,0 ha, localizada aproximadamente $230 \mathrm{~m}$ da área MA, é um pequeno remanescente de Floresta Estacional Semidecidual (Floresta Tropical Atlântica) existente na região, constituída de vegetação típica do bioma da Mata Atlântica, sendo a área que mais se aproxima do estado natural. O Campo Naturalizado (CN; 28²1149.99”'S, 49²7'23.96”'W), apresenta cerca de 6,0 ha, localizada aproximadamente $230 \mathrm{~m}$ da área MA, considerada como campo naturalizado após a retirada da mata em anos anteriores para utilização com pastagem, porém na época das coletas encontrava-se, de acordo com o proprietário, em pousio de médio prazo (período de pousio $>1-2$ anos), sendo composta por espécies de gramíneas, leguminosas com predominância de capimlimão (Cymbopogon sp.) e pega-pega (Desmodium sp.) e outras espécies nativas ou naturalizadas.

Para coleta da mesofauna utilizou-se um cilindro de aço inoxidável de $17 \mathrm{~cm}$ de diâmetro, amostrando o solo na profundidade $5 \mathrm{~cm}$, com volume de solo de 1134,9 $\mathrm{cm}^{3}$, sendo coletadas seis amostras em um transecto com distância de $10 \mathrm{~m}$ entre pontos. O solo retirado foi acondicionado em sacos plásticos para minimizar as perdas de umidade, identificados e levados para o Laboratório de Microbiologia e Fauna do Solo, do Departamento de Solos e Recursos Naturais do Centro de Ciências Agroveterinárias da Universidade do Estado de Santa Catarina. A extração da mesofauna foi utilizada o método de Berllese modificado (SOUTHWOOD, 1968), onde o solo permaneceu por um período de três dias. Após o período de exposição, as amostras dos frascos foram passadas em peneiras com malhas de 0,20, 0,15 e $0,10 \mathrm{~mm}$, para separar o solo e os fragmentos vegetais.

Os organismos extraídos das amostras foram colocados em potes plásticos e conservados em solução de álcool (70\%) para posterior identificação e quantificação, com auxílio de uma lupa binocular de aumento $40 \mathrm{x}$. Assim, os insetos imaturos e adultos coletados foram identificados quanto à Ordem [Coleoptera (Colp); Dermaptera (Der); Diptera (Dipt); Hymenoptera, especificamente a família Formicidae (For); Heteroptera - Hemiptera (Het); Isoptera (Ispt); Larva de Coleoptera + Pupa de Coleoptera (LCol); Larva de Diptera (LDipt); Orthoptera (Ort); Thysanoptera (Thy)], com auxílio de literatura específica (GALLO et al., 2002; COSTA et al., 2006), o restante dos organismos invertebrados foram classificados em grupos taxonômicos: Arachnida [Acari (Aca), Araneae (Ara), Pseudoscorpionida (Pse)], Collembola (Coll), Diplura (Dipl), Enchytraeidae (Enc), Isopoda (Isop), Myriapoda [Chilopoda (Chi), Diplopoda (Dip), Symphyla (Sym)], Protura (Pro) e Outros Grupos (OG; organismos não identificados e grupos de menor ocorrência) (BRUSCA; BRUSCA, 2003).

As análises químicas do solo foram realizadas em amostras coletadas, na profundidade de $5 \mathrm{~cm}$ com trado de rosca, ao lado dos pontos amostrais para avaliação da mesofauna. Os atributos químicos analisados foram pH em água, $\mathrm{Ca}, \mathrm{Mg}, \mathrm{Al}, \mathrm{P}, \mathrm{K}$ e matéria orgânica (MO) (Tabela 1), de acordo com o protocolo descrito por Tedesco et al. (1995). Ainda, ao lado dos pontos amostrais, foi coletada uma amostra para quantificação do teor de umidade (US) e medida a temperatura do solo (TS). Também foram obtidas informações sobre a precipitação total e temperatura do ar (mínima, média 
TABELA 1: Características químicas (média \pm desvio padrão) do solo na profundidade de $5 \mathrm{~cm}$ nas áreas estudadas da Mina A3, da Mina A5, da Mata Nativa e do Campo Naturalizado, no município de Lauro Müller/SC.

\begin{tabular}{|c|c|c|c|c|c|c|c|}
\hline \multirow{2}{*}{ Área* } & pH & $\mathrm{Ca}^{2+}$ & $\mathrm{Mg}^{2+}$ & $\mathbf{A l}^{3+}$ & $\mathbf{P}_{\text {Mehlich }}$ & \multirow{2}{*}{$\frac{\mathbf{K}^{+}}{\mathrm{mg} \mathrm{dm^{-3 }}}$} & \multirow{2}{*}{$\begin{array}{c}\text { MO } \\
\% \\
\end{array}$} \\
\hline & $\mathrm{H}_{2} \mathrm{O}$ & \multicolumn{4}{|c|}{----------- $\mathrm{cmol}_{\mathrm{c}} \mathrm{dm}^{-3}$--------- } & & \\
\hline $\mathrm{A} 3(n=24)$ & $6,3 \pm 0,2$ & $4,14 \pm 2,63$ & $2,65 \pm 2,33$ & $0,18 \pm 0,13$ & $46,75 \pm 22,48$ & $206 \pm 96$ & $2,20 \pm 0,16$ \\
\hline $\mathrm{A} 5(n=24)$ & $5,4 \pm 0,1$ & $3,11 \pm 1,89$ & $1,59 \pm 1,23$ & $0,43 \pm 0,40$ & $25,38 \pm 11,38$ & $125 \pm 37$ & $3,73 \pm 0,13$ \\
\hline $\mathrm{MN}(n=24)$ & $3,6 \pm 0,2$ & $1,35 \pm 1,27$ & $1,41 \pm 1,24$ & $5,48 \pm 0,31$ & $9,08 \pm 1,82$ & $117 \pm 36$ & $3,63 \pm 0,36$ \\
\hline $\mathrm{CN}(n=24)$ & $4,1 \pm 0,1$ & $3,06 \pm 1,48$ & $1,16 \pm 0,24$ & $3,65 \pm 0,72$ & $3,88 \pm 2,63$ & $127 \pm 142$ & $3,13 \pm 0,15$ \\
\hline
\end{tabular}

*A3: Mina A3; A5: Mina A5; MN: Mata Nativa; CN: Campo Naturalizado. Médias das quatro épocas de coleta ( \pm desvio padrão).

e máxima) da região da EPAGRI/CIRAM/INMET da Estação Urussanga/SC, distante aproximadamente 16 km de Lauro Müller.

A partir do resultado de densidade de indivíduos (ind. $\mathrm{m}^{-2}$ ), foi obtida a frequência relativa e a riqueza média dos grupos taxonômicos. O tratamento estatístico dos dados foi realizado por dois métodos. $\mathrm{O}$ primeiro método consistiu de uma análise de variância (ANOVA) e as médias comparadas por meio do teste LSD $(p<0,05)$, utilizando o Software Statistica 7.0 (STATSOFT, 2004), dos atributos da fauna do solo (densidade e riqueza). O segundo método consistiu em uma análise multivariada dos dados para diferenciação das áreas.

A curva de resposta principal ("Principal Response Curve" - PRC) (BRINK; TER BRAAK, 1999) é um método de ordenação conhecido como análise de redundância parcial ( $p$ RDA), indicada para avaliar o efeito de um determinado fator sobre uma comunidade ao longo do tempo. Este método possibilita resumir os efeitos de cada tratamento em relação ao controle (Mata Nativa) sobre os grupos da fauna do solo. A PRC extrai informação de apenas parte da variância que é explicada pelo fator empregado como tratamento (áreas) e o tempo (épocas de amostragem), que é utilizado como co-variável (LEPŠ; ŠMILAUER, 2003). Na PRC, o tempo é disposto no eixo $\mathrm{X}$ e coeficiente canônico, também denominado como coeficiente canônico padronizado, relativo ao tratamento controle, no eixo Y. Os coeficientes canônicos dos demais tratamentos são apresentados como desvios em relação ao tratamento controle, para cada tempo avaliado.
Junto ao gráfico da PRC é apresentado um diagrama, no qual estão plotados os pesos (valores positivos ou negativos) dos grupos da fauna do solo avaliados. São obtidos uma razão $\mathrm{F}$ e um valor $p$ de significância determinados por meio do teste de permutação de Monte Carlo através do Software CANOCO 4.5 (TER BRAAK; SMILAUER, 1998). Para avaliar diferenças entre os tratamentos em relação ao controle, foi empregado um teste de homogeneidade (teste do Chi quadrado de Bartlett) e em seguida um teste "post-hoc" de Dunnett de duas caudas, utilizando o Software Statistica 7.0 (STATSOFT, 2004).

\section{Resultados e Discussão}

\section{Densidade, riqueza e frequência relativa}

Verificou-se que houve efeito significativo ( $p<$ $0,05)$ entre as épocas de coleta da fauna do solo nas áreas A3, A5, MN e CN (Tabela 2). Os valores de densidade variaram de 396 ind. $\mathrm{m}^{-2}$ na área de MN na Época 4 de coleta a 12.173 ind. $\mathrm{m}^{-2}$ na área de $\mathrm{CN}$ na Época 1 . Ainda, constatou-se que houve efeito significativo $(p<0,05)$ entre as áreas durante a mesma época somente na Época 2, onde as áreas de $\mathrm{CN}$ e $\mathrm{MN}$ apresentaram os maiores valores de densidade. Na Época 1, a área de $\mathrm{CN}$ não diferiu das áreas A3, A5 e MN. Na Época 3, a área MN não diferiu das áreas $\mathrm{CN}$, $\mathrm{A} 5$ e A3; e na Época 4, a área $\mathrm{A} 3$ não diferiu das áreas $\mathrm{CN}, \mathrm{A} 5 \mathrm{e} \mathrm{MN}$. 
TABELA 2: Densidade média de organismos (ind. $\mathrm{m}^{-2}$ ) e riqueza média da comunidade da fauna invertebrada do solo coletadas na Mina A3, Mina A5, Mata Nativa (MN) e no Campo Naturalizado (CN). Média \pm desvio padrão.

\begin{tabular}{lcccc}
\hline \multicolumn{1}{c}{ Épocas } & $\mathbf{A 3}$ & $\mathbf{A 5}$ & $\mathbf{M N}$ & $\mathbf{C N}$ \\
\hline & & \multicolumn{2}{c}{ Densidade média de organismos } \\
Época 1 (out/09) & $10.044 \pm 7.317 \mathrm{aA}$ & $9.853 \pm 6.404 \mathrm{aA}$ & $5.235 \pm 3.205 \mathrm{aA}$ & $12.173 \pm 6.993 \mathrm{aA}$ \\
Época 2 (fev/10) & $1.109 \pm 1.074 \mathrm{bB}$ & $3.987 \pm 3.781 \mathrm{bB}$ & $5.705 \pm 5.552 \mathrm{aA}$ & $11.079 \pm 8.985 \mathrm{aA}$ \\
Época 3 (mai/10) & $1.197 \pm 790 \mathrm{bA}$ & $2.445 \pm 1.999 \mathrm{bA}$ & $4.883 \pm 5.482 \mathrm{aA}$ & $2.034 \pm 2.117 \mathrm{bA}$ \\
Época 4 (ago/10) & $1.050 \pm 707 \mathrm{bA}$ & $690 \pm 434 \mathrm{bA}$ & $396 \pm 252 \mathrm{bA}$ & $925 \pm 547 \mathrm{bA}$ \\
\hline \multicolumn{5}{c}{ Riqueza média } \\
Época 1 (out/09) & $7,5 \pm 0,5 \mathrm{aA}$ & $5,8 \pm 1,5 \mathrm{aB}$ & $7,0 \pm 1,1 \mathrm{aAB}$ & $8,2 \pm 1,2 \mathrm{aA}$ \\
Época 2 (fev/10) & $4,3 \pm 2,3 \mathrm{bA}$ & $3,5 \pm 1,5 \mathrm{bA}$ & $6,8 \pm 3,4 \mathrm{aA}$ & $6,2 \pm 1,2 \mathrm{bA}$ \\
Época 3 (mai/10) & $4,0 \pm 1,7 \mathrm{bA}$ & $4,2 \pm 1,5 \mathrm{abA}$ & $5,2 \pm 2,8 \mathrm{aA}$ & $5,3 \pm 1,5 \mathrm{bA}$ \\
Época 4 (ago/10) & $2,5 \pm 1,4 \mathrm{bA}$ & $2,7 \pm 1,2 \mathrm{bA}$ & $2,2 \pm 1,3 \mathrm{bA}$ & $2,7 \pm 1,0 \mathrm{cA}$ \\
\hline
\end{tabular}

Letras minúsculas na coluna representam diferenças entre épocas e letras maiúsculas na linha representam diferenças entre os tratamentos, diferem significativamente entre si pelo teste LSD $(p<0,05)$.

Os valores de riqueza dos grupos taxonômicos diferiram entre as épocas de coleta para áreas A3, $\mathrm{A} 5, \mathrm{MN}$ e $\mathrm{CN}$, com maior valor de riqueza na época mais quente (Época 1 e 2), ocorrendo uma diminuição da riqueza de grupos na época mais fria (Épocas 3 e 4; Tabela 2). Em relação à ocorrência de diferenças significativas $(p<0,05)$ entre as áreas durante a mesma época de coleta, somente a Época 1 apresentou diferenças entre as áreas, em que as áreas $\mathrm{CN}$ e A3 apresentaram os maiores valores.

Comunidades do solo são variáveis ao longo do tempo (SCHON et al., 2012) e respondem às alterações nas condições climáticas, como a temperatura do ar e a precipitação pluviométrica que apresentam enorme influência na temperatura e umidade do solo, que de acordo com Han et al. (2007), são fatores críticos que regulam a atividade biológica nos solos, como a presença/ausência e mobilidade/imobilidade de espécies da fauna do solo.

Nesse experimento, as temperaturas médias do ar no período das coletas foram de 19, 26, 18 e $15^{\circ} \mathrm{C}$ nas Épocas 1, 2, 3 e 4, respectivamente, e a precipitação total mensal foi de 82, 160, 374 e $73 \mathrm{~mm}$ nas Épocas 1, 2, 3 e 4, respectivamente. À medida que a atividade de invertebrados depende da temperatura e umidade, diferenças de valores de densidade e riqueza de organismos entre as épocas de coleta devem, provavelmente, à variação climática. Já que o incremento da temperatura e da precipitação na primeira e segunda coleta pode ter possibilitado um aumento no crescimento de plantas e com isso na disponibilidade de alimento. Já as temperaturas mais baixas, nos meses de Maio (Época 3) e Agosto (Época 4), podem ser a explicação para a menor densidade da fauna daquele período.

Ao total foram encontrados 19 táxons nas áreas durante o período de coleta na região de Lauro Müller (Tabela 3). Dentre os grupos mais frequentes da comunidade da fauna do solo encontrados nas áreas destacam-se Acari, Collembola e Formicidae que apresentaram as maiores densidades, independente da área e época de coleta, com frequência relativa diferenciada destes grupos em cada época.

Observar a evidência de que a área de $\mathrm{MN}$ apresentou uma maior riqueza total de grupos taxonômicos que as demais áreas, considerando as quatro épocas de coleta pode estar relacionada ao fato de áreas naturais apresentarem uma maior diversidade vegetal, fazendo com que tenha maior disponibilidade de alimento. De acordo com Frouz et al. (2008), as plantas desempenham um papel principal na formação da estrutura física do habitat, além de fazerem parte da cadeia alimentar.

Ainda, a área $\mathrm{MN}$ foi a única área a ter sido coletado o grupo Pseudoscorpionida, embora com uma baixa frequência relativa $(0,23 \%)$, pois normalmente esse 
grupo só é encontrado em ambientes cujo ecossistema não apresenta degradação. Segundo Yamamoto et al. (1994), os Pseudoscorpionida são bons bioindicadores, já que são sensíveis a ações antrópicas e encontram-se em maiores densidades populacionais em ambientes com maior equilíbrio ecológico.

TABELA 3: Grupos taxonômicos da comunidade da fauna invertebrada do solo coletados durante o experimento.

\begin{tabular}{clc}
\hline Área* & \multicolumn{1}{c}{ Grupos taxonômicos** } & Total \\
\hline A3 & $\begin{array}{l}\text { Aca, Ara, Chi, Coll, Colp, Dipt, Enc, For, } \\
\text { Het, Isop, Ort, Sym }\end{array}$ & 12 \\
\hline A5 & $\begin{array}{l}\text { Aca, Ara, Chi, Coll, Colp, Der, Dipl, Dipt, } \\
\text { Enc, For, Het, Isop, Pro, Sym }\end{array}$ & 14 \\
\hline MN & $\begin{array}{l}\text { Aca, Ara, Chi, Coll, Colp, Der, Dip, Dipt, } \\
\text { Enc, For, Het, Isop, Pro, Pse, Sym, Thy }\end{array}$ & 16 \\
\hline CN & $\begin{array}{l}\text { Aca, Ara, Chi, Coll, Colp, Dipt, Enc, For, } \\
\text { Het, Isop, Ispt, Pro, Sym, Thy }\end{array}$ & 14 \\
\hline
\end{tabular}

*A3 - Área A3, A5 - Área A5, MN - Mata Nativa e CN - Campo Naturalizado. **Aca: Acari; Ara: Araneae; Chil: Chilopoda; Coll: Collembola; Colp: Coleoptera; Der: Dermaptera; Dip: Diplopoda; Dipl: Diplura; Dipt: Diptera; Enc: Enchytraeidae; For: Formicidae; Het: Heteroptera - Hemiptera; Isop: Isopoda; Ispt: Isoptera; Ort: Orthoptera (Paquinha); Pro: Protura; Pse: Pseudoscorpionida; Sym: Symphyla; Thy: Thysanoptera.
A diversidade de grupos taxonômicos também foi expressa pela frequência relativa (FR) dos grupos (Figura 1). Considerando as quatro épocas juntas, os grupos que apresentaram maior FR foram os ácaros que tiverem maior ocorrência nas áreas $\mathrm{CN}(44 \%), \mathrm{MN}$ (35\%), A5 (23\%) e A3 (20\%), os colêmbolos na área A5 (61\%), A3 (28\%), MN (20\%) e CN (11\%) e as formigas na área CN (33), MN (27\%) e A3 (13\%). Os demais grupos apresentaram FR menor que 5\% em todas as áreas e épocas de coleta.

Cabe observar que a área A3 apresentou uma maior frequência relativa dos grupos Heteroptera-Hemiptera $(25 \%)$ e de Diptera (6\%) em relação às demais áreas, provavelmente em função dessa área ser de uso agrícola e proporcionar o surgimento de nichos para essas espécies. Segundo Bröring e Wiegleb (2005), o grupo Heteroptera - Hemiptera (percevejos), que compreende insetos zoofagos e fitófagos, habitam a camada superficial do solo e o estrato herbáceo, sendo que o número de organismos é fortemente influenciado pela composição da vegetação local como da área circundante.

FIGURA 1: Frequência relativa (\%) de grupos da fauna invertebrada do solo coletadas na Mata Nativa (MN), no Campo Naturalizado (CN), na Área A3 (A3) e na Área A5 (A5), independente da época de coleta. *Aca: Acari; Coll: Collembola; Dipt: Diptera; For: Formicidae; Het: Heteroptera - Hemiptera; LDipt: Larva de Diptera; OG: outros grupos (organismos não identificados e com frequência relativa menor que 5). Média de seis repetições e quatro épocas de coleta $(n=6 \times 4)$.

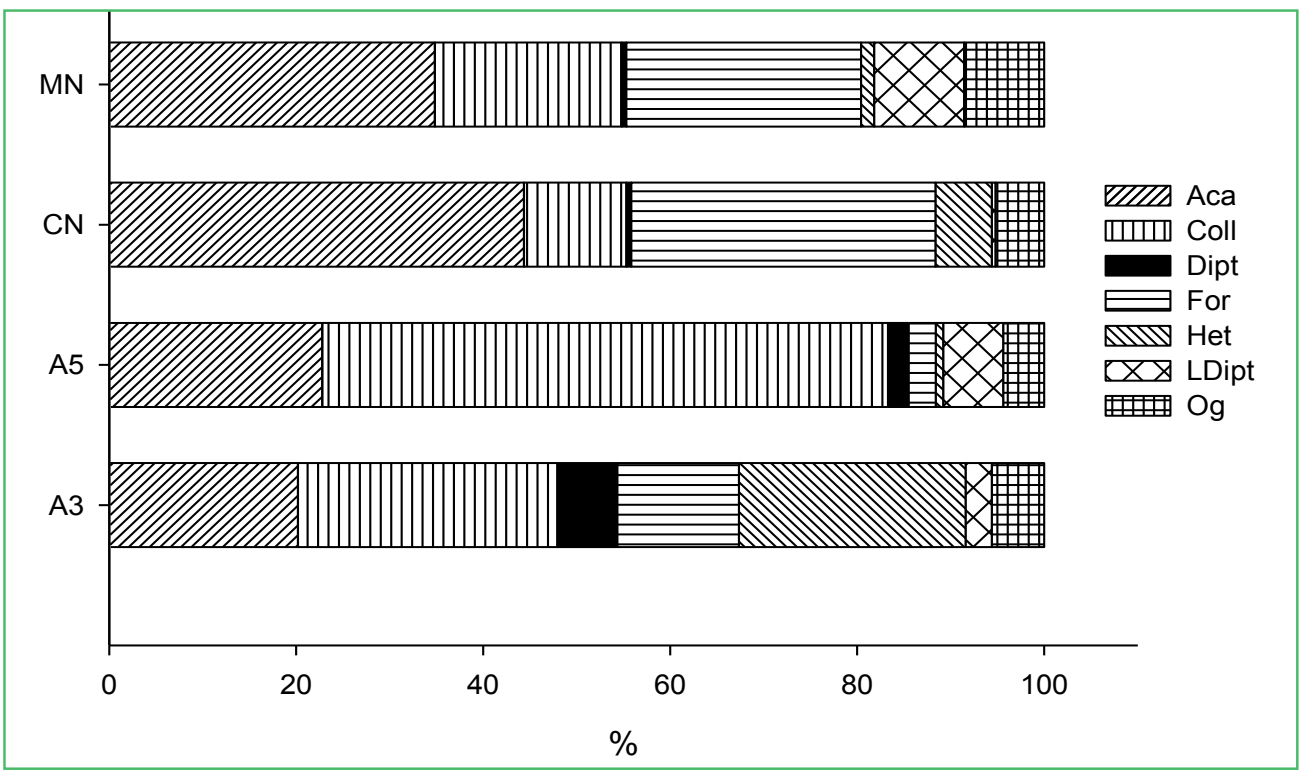


Ainda, a área $\mathrm{MN}$ apresentou maior frequência relativa de larvas de dípteras $(10 \%)$. De acordo com Frouz (1999) e Coleman et al. (2004), as larvas de dípteras participam de processos biológicos importantes no solo, como decomposição da matéria orgânica e ciclagem de nutrientes. Em alguns ecossistemas, representam os organismos mais abundantes em função de fatores favoráveis como maior camada de serapilheira e umidade (FROUZ, 1999), fatores esses que podem ser interpretados pelos altos valores de matéria orgânica (Tabela 1) e umidade (dados não apresentados) na $\mathrm{MN}$, podendo ser um importante local para reprodução desse grupo.

A análise de variância dos atributos da fauna (densidade e riqueza) revela que as áreas antropizadas promovem um ambiente adequado para a composição de grupos da mesofauna edáfica em relação aos grupos encontrados na Mata Nativa (controle). Ou seja, o campo naturalizado, a Mina A5 e a Mina A3 apresentaram grupos taxonômicos semelhantes ao controle, satisfazendo os serviços do ecossistema prestados por eles, podendo indicar a qualidade dos ecossistemas e, consequentemente, o sucesso de projetos de recuperação/restauração.

\section{Curva de resposta principal}

A análise da curva de resposta principal (PRC) calculada para as áreas de Lauro Müller (Figura 2) revelou que $20,2 \%$ da variância da abundância da fauna é explicada pelo tempo e $23,4 \%$ pelas áreas. O primeiro eixo canônico da PRC capturou uma parte significativa (44,8\%) da variância explicada pelas áreas (teste de permutação de Monte Carlo, 999 permutações, $p=$ 0,002). Em todas as Épocas de amostragem ocorreu diferença entre a Mata Nativa (controle) e no mínimo em uma das áreas antropizadas (Campo Naturalizado, Área A3 e Área A5) diferiram significativamente da Mata Nativa (teste de Dunnett; two-side, $p<0,01$ ).

A análise PRC fornece para cada grupo da fauna um valor de peso, sendo os maiores valores tanto positivos quanto negativos, correspondentes aos grupos que mais foram influenciados pelos tratamentos. Os grupos que tiveram os maiores pesos calculados foram Formicidae
$(+1,23)$ seguido de Acari $(+0,59)$, enquanto o grupo Diptera (adultos e larvas) apresentou o maior peso negativo $(-0,39)$. Valores positivos maiores indicam maior sensibilidade do grupo ao longo do tempo em comparação a área controle (Mata Nativa). Estes grupos indicam uma maior contribuição para a variação. Estes grupos foram as mais afetados em termos de abundância, enquanto que o restante dos demais grupos não foram tão inlfuenciados. Isto pode ser atribuído às diferentes exigências ecológicas e estratégias de vida de cada grupo.

A área $\mathrm{CN}$ apresentou maior abundância dos grupos Formicidae, Acari, Araneae, Isopoda e Isoptera em comparação as demais áreas, independente da época de coleta. Por outro lado a área de $\mathrm{CN}$ apresentou grupos que não foram diferentes das demais áreas (próximos a zero) como Chilopoda, Coleoptera (adultos e larvas) e Protura, e menores valores de abundância para os grupos Dermapetra, Diplopoda, Diplura, Enchytraeidae, Heteroptera - Hemiptera, Hymenotpera, Orthopetra e Pseudoscorpionida.

Cabe observar ainda que possivelmente à medida que aumenta a colonização da fauna edáfica as áreas vão atingindo um padrão próximo ou superior ao da $\mathrm{MN}$, possível de se observar em todas as épocas para a área $\mathrm{CN}$ e nas épocas de maio e agosto de 2010 para a área A3, a qual se aproximou muito da mata nativa.

Considerando a análise da comunidade da fauna edáfica como um todo, verificou-se que a riqueza de grupos não foi influenciada pelo processo de construção do solo após a mineração de carvão.

A época de amostragem e a construção do solo após a mineração de carvão não influenciaram a abundância de grupos da fauna do solo.

A construção do solo após a mineração de carvão influenciou somente alguns grupos da fauna do solo, especialmente Formicidae, Acari e Diptera (adultos e larvas), independente da época de coleta.

A curva de resposta principal revela diferenças na reação dos diferentes grupos para as áreas antropizadas e sua utilização é uma importante ferramenta de análise estatística multivariada, destacando os grupos da fauna do solo que mais contribuem na separação das áreas. 
FIGURA 2: Curvas de resposta principal de solos da Mina 3 (A3), Mina 5 (A5), Campo Naturalizado (CN) e Mata (MN) no período de outubro de 2009 a agosto de 2010 sobre a abundância de grupos da fauna do solo (Cdt - Coeficiente canônico padronizado da RDA parcial) e o peso dos grupos da fauna do solo (Bk), no município de Lauro Müller/SC. Juntamente é apresentado o quadro da análise estatística.

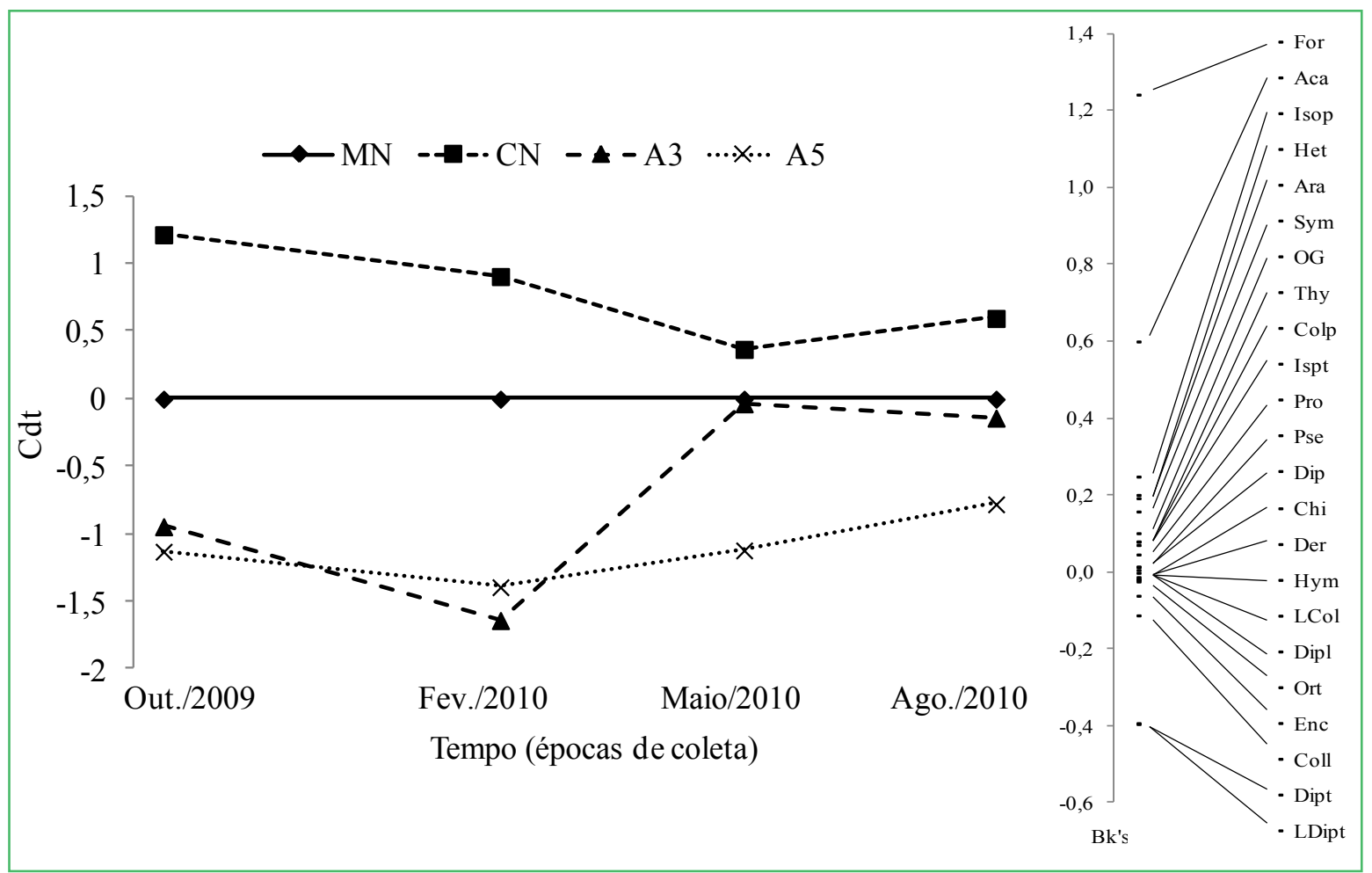

\begin{tabular}{|c|c|c|c|c|c|c|c|}
\hline \multicolumn{3}{|c|}{$\begin{array}{l}\text { Teste de permutação de Monte Carlo para } \\
\text { significância do 1o eixo canônico da PRC }\end{array}$} & \multicolumn{2}{|c|}{$\begin{array}{l}\text { \% da variância } \\
\text { explicada pelo }\end{array}$} & \multicolumn{3}{|c|}{$\begin{array}{l}\text { \% da variância explicada pelas áreas avaliada } \\
\text { pelo } 1^{\text {o }} \text { eixo canônico da PRC }\end{array}$} \\
\hline \multicolumn{2}{|c|}{$\begin{array}{l}\text { Autovalor }=0,105 \\
F=13,919\end{array}$} & $\begin{array}{l}p=0,002 \\
\text { Conteúdo explicativo }=20,2\end{array}$ & 20,2 & 23,4 & \multicolumn{3}{|c|}{$44,8 \%$} \\
\hline \multirow{2}{*}{ Épocas } & \multicolumn{2}{|r|}{ Homogeneidade } & \multicolumn{5}{|c|}{ Teste de Dunnett (two-side) } \\
\hline & Bartlett & $\mathrm{p}$ & $\mathrm{F}$ & $p$ & $\mathrm{MN}$ & A5 & $\mathrm{CN}$ \\
\hline E1 & 0,58 & 0,902 & 11,21 & 0,00016 & $\mathbf{0 , 0 4}$ & 0,05 & $\mathbf{0 , 0 3}$ \\
\hline E2 & 1,51 & 0,681 & 10,99 & 0,00018 & 0,01 & $\mathbf{0 , 0 3}$ & 0,21 \\
\hline E3 & 7,65 & 0,054 & 4,55 & 0,01378 & 0,99 & 0,04 & 0,72 \\
\hline E4 & 5,22 & 0,156 & 5,64 & 0,00571 & 0,95 & 0,04 & 0,21 \\
\hline
\end{tabular}

\section{Referências}

ALBUQUerQue, J. A.; ALMEIDA, J. A.; GATIBONI, L. C.; ELTZ, F. L. F. Atividades agrícolas de produção em solos frágeis no sul do Brasil. Tópicos em Ciência do Solo, Viçosa, v. 7, p. 367403, 2011.

ANDREOLA, A. Avaliação dos atributos químicos de um solo construído pós-mineração de carvão no município de Lauro Müller, SC. 2011. 71 f. Dissertação (Mestrado em Manejo do Solo) - Universidade do Estado de Santa Catarina, Lages. 2011.

BRINK, P. J.; TER BRAAK, C. J. F. Principal response curves: analysis of time-dependent multivariate responses of biological community to stress. Environmental Toxicology and Chemistry, Houston, v. 18, p. 138-148, 1999.

BRÖRING, U.; WIEGLEB, G. Soil zoology II: Colonization, distribution, and abundance of terrestrial Heteroptera in open landscapes of former brown coal mining areas. Ecological Engineering, Columbus, v. 24, p. 135-147, 2005.

BRUSCA, R. C.; BRUSCA, G. J. Invertebrates. Sunderland: Sinauer Associates, 2 ed. 2003. 938 p.

CAMPOS, M. L.; ALMEIDA, J. A.; SOUZA, L. S. Avaliação de três áreas de solo construído após mineração de carvão a céu aberto em Lauro Müller, Santa Catarina. Revista Brasileira de Ciência do Solo, Viçosa v. 27, p. 1123-1137, 2003. 
CARDOSO, P.; ERWIN, T. L.; BORGES, P. A. V.; NEW, T. R. The seven impediments in invertebrate conservation and how to overcome them. Biological Conservation, Boston, v. 144, p. $2647-$ $2655,2011$.

CASTILHOS, Z. C.; FERNANDES, F. R. C. A bacia carbonífera sul catarinense e os impactos e passivos da atividade da indústria extrativa mineral de carvão na territorialidade. In: FERNANDES, F. R. C.; ENRÍQUEZ, M. A. R. S.; ALAMINO, R. C. J. (Ed.). Recursos minerais \& sustentabilidade territorial: grandes minas. Rio de Janeiro: CETEM/MCTI, 2011. p. 361-386.

COLEMAN, D. C.; CROSSlEY JR., D. A.; HENDRIX, P. F. Fundamentals of soil ecology. 2. ed. Amsterdam: Elsevier Academic Press, 2004. 404 p.

CORREIA, M. E. F. Relações entre a diversidade da fauna de solo e o processo de decomposição e seus reflexos sobre a estabilidade dos ecossistemas. Documentos $\mathrm{n}^{\circ}$ 156. Seropédica: Embrapa Agrobiologia, 2002. 18 p.

COSTA, C.; IDE, S.; SIMONKA, C. E. Insetos imaturos. Metamorfose e identificação. Ribeirão Preto: Editora Holos, 2006. 249 p.

EMBRAPA - EMPRESA BRASILEIRA DE PESQUISA AGROPECUÁRIA. Sistema brasileiro de classificação de solos. 2 ed. Brasília: Centro Nacional de Pesquisa de Solos, 2006. 306 p.

FROUZ, J. Use of soil dwelling Diptera (Insecta, Diptera) as bioindicators: a review of ecological requirements and response to disturbance. Agriculture, Ecosystems and Environment, Amsterdam, v. 74, p. 167-186, 1999.

FROUZ, J.; ELHOTTOVÁ, D.; KURÁŽ, V.; ŠOURKOVA, M. Effects of soil macrofauna on other soil biota and soil formation in reclaimed and unreclaimed post mining sites: Results of a field microcosm experiment. Applied Soil Ecology, Amsterdam, v. 33, p. 308-320, 2006.

FROUZ, J.; PRACH, K.; PIŽL, V.; HÁNĚL, L.; STARÝ, J.; TAJOVSKÝ, K.; MATERNA, J.; BALÍK, V.; KALČÍK, J.; ŘEHOUNKOVÁ, K. Interactions between soil development, vegetation and soil fauna during spontaneous succession in post mining sites. European Journal of Soil Biology, Braunschweig, v. 44, p. 109-121, 2008.

GALLO, D.; NAKANO, O.; NETO, S. S.; CARVALHO, R. P. L.; BATISTA, G. C.; FILHO, E. B.; PARRA, J. R. P.; ZUCCHI, R. A.; ALVES, S. B.; VENDRAMIM, J. D.; MARCHINI, L. C.; LOPES, J. R. S.; OMOTO, C. Entomologia agrícola. Piracicaba: FEALQ, 2002. 920 p.
HAN, G.; ZHOU, G.; XU, Z.; YANG, Y.; LIU, J.; SHI, K. Biotic and abiotic factors controlling the spatial and temporal variation of soil respiration in an agricultural ecosystem. Soil Biology and Biochemstry, Oxford, v. 39, p. 418-425, 2007.

LEPŠ, J.; ŠMILAUER, P. Multivariate analysis of ecological data using CANOCO. Cambridge: Cambridge University Press, 2003. 269 p.

LUNARDI NETO, A.; ALBUQUERQUE, J. A.; ALMEIDA J. A.; MAFRA, A. L.; MEDEIROS, J. C.; ALBERTON, A. Atributos físicos do solo em área de mineração de carvão influenciados pela correção da acidez, adubação orgânica e revegetação. Revista Brasileira de Ciência do Solo, Viçosa, v. 32, p. 1379-1388, 2008.

NICHOLS, O. G.; NICHOLS, F. M. Long-term trends in faunal recolonization after bauxite mining in the Jarrah forest of southwestern Australia. Restoration Ecology, Crawley, v. 11, p. 261-272, 2003.

SCHON, N. L.; MACKAY, A. D.; MINOR, M. A. Vulnerability of soil invertebrate communities to the influences of livestock in three grasslands. Applied Soil Ecology, Amsterdam, v. 53, p. 98-107, 2012.

SOUTHWOOD, T. R. E Ecological methods with particular reference to the study of insect populations. London: Chapman \& Hall, 1968. 391 p.

STATSOFT, Inc. STATISTICA (Data analysis software system). Version 7, 2004. Disponível em: <www.statsoft.com>.

TEDESCO, M. J.; GIANELLO, C.; BISSANI, C. A.; BOHNEN, H.; VOLKWEISS, S. J. Análises de solo, plantas e outros materiais. 2. ed. Porto Alegre: UFRGS, 1995. 174 p. (Boletim Técnico de Solos, 5).

TER BRAAK, C. J. F.; SMILAUER, P. CANOCO reference manual and user's guide to Canoco for Windows: software for canonical community ordination (Version 4). Ithaca: Microcomputer Power, 1998. 352 p.

YAMAMOTO, T.; TOUYAMA, Y.; NAKAMURA, K.; HIDAKA, K.; TAKAHASHI, F. Ecological study of forest floor invertebrate fauna in naturally regenerated cedar forest and cedar plantations. Edaphologia, Yokohama, v. 51, p. 19-32, 1994.

ZEPPELINI, D.; BELLINI, B. C.; CREÃO-DUARTE, A. J.; HERNÁNDEZ, M. I. M. Collembola as bioindicators of restoration in mined sand dunes of Northeastern Brazil. Biodiversity and Conservation, New York v. 18, p. 1161-1170, 2009. 